\title{
ARTÍCULOS
}

Recibido 01.08.2012. Aprobado 23.10.2012

Evaluado por el sistema double blind review. Editor Científico Max Fortunato Cohen

\section{EL EFECTO DE LA TRIPLE HÉLICE EN LOS RESULTADOS DE INNOVACIÓN}

\author{
Efeito da hélice tríplice em desempenho de inovação \\ The triple helix effect on innovation performance
}

\begin{abstract}
RESUMEN
La innovación está relacionada con el crecimiento de las economías y de las empresas, y en un entorno cambiante como el actual, se presenta como una herramienta fundamental para conseguir ventajas competitivas. Esta investigación tiene por objetivo analizar la relación existente entre la capacidad de innovación de las empresas y la obtención de información de las mismas a través de la cooperación con competidores, proveedores, Universidades y otras instituciones públicas (Triple Hélice). En primer lugar se desarrolla una revisión bibliográfica de los conceptos relacionados para posteriormente, a través del método de ecuaciones estructurales, dar respuesta y aceptar la hipótesis propuesta que pone de manifiesto la relación existente entre los resultados de innovación de las empresas y la información obtenida de la Triple Hélice. La investigación se realiza con una muestra de empresas españolas, encuestadas por el Instituto Nacional de Estadística (INE), durante los años 2008-2010.
\end{abstract}

PALABRAS CLAVE I Innovación, triple hélice, universidad, empresa, gobierno.

\section{RESUMO}

A inovação está relacionada ao crescimento das economias e das empresas, e em um ambiente em mudança como o presente, é apresentado como uma ferramenta fundamental para a vantagem competitiva. Esta pesquisa visa analisar a relação entre a capacidade de inovação das empresas e a obtenção da mesma informação por meio da cooperação com concorrentes, fornecedores, universidades e outras instituições públicas (Hélice Tríplice). Primeiro, nós desenvolvemos uma revisão da literatura sobre conceitos relacionados e, em seguida, por meio do método de equações estruturais, um modelo para responder e aceitar a hipótese proposta que destaca a relação entre o desempenho inovador das empresas e informações obtidas a partir da Hélice Tríplice. A pesquisa é realizada com uma amostra de empresas espanholas pesquisadas pelo Instituto Nacional de Estatística (INE), durante os anos 2008-2010.

\section{MARÍA JESÚS LUENGO}

mariajesus.luengo@ehu.es

Profesora do Departamento de Evaluación de la Gestión e Innovación Empresarial, Universidad del País Vasco/Euskal Herriko Unibertsitatea - Bilbao - País

Vasco, España

\section{MARÍA OBESO}

maria.obeso@unican.es Profesora do Departamento de Administración de Empresas, Universidad de Cantabria Santander - Cantabria, España
PALAVRAS-CHAVE | Inovação, hélice tríplice, universidade, empresa, governo.

\section{ABSTRACT}

Innovation is related to the growth of economies and companies, and in a changing environment, like the present, it is introduced as a fundamental means for competitive advantage. This study aims at analyzing the relation between the companies' capacity for innovation and obtaining the same information by means of cooperation with competitors, suppliers, universities and public institutions (Triple Helix). First, we developed a literature review on related concepts and, then, by using the structural equation method, we elaborated a model to respond to and accept the hypothesis proposed, which highlights the relation between the innovative performance of companies and the information obtained from the Triple Helix. The survey was conducted with a sample of Spanish companies researched by the National Institute of Statistics (INE) from 2008 to 2010.

KEYWORDS / Innovation, Triple helix, university, company, government. 


\section{INTRODUCCIÓN}

Desde la década de los 90, nos encontramos inmersos la sociedad del conocimiento (SAKAIYA, 1991), donde la innovación se convierte en una herramienta fundamental para la consecución de ventajas competitivas. Se entiende por sistema de innovación al conjunto de reglas y procedimientos que incrementan la adquisición, la creación y el uso del conocimiento (CHEN e DALHMAN, 2004).

En una sociedad como la actual, donde los cambios se suceden ininterrumpidamente, la innovación y el conocimiento se convierten en los principales motores de la economía (VASCONCELOS y otros, 2008). La Organización para la Cooperación y el Desarrollo Económico (OCDE) sostiene que la innovación es el motor de crecimiento de los países desarrollados, atribuyéndole cerca del 50 por ciento del mismo (ROOS e PIKE, 2011).

Sobre eso, presentamos un estudio que relaciona los resultados de innovación de las empresas con el modelo Triple Hélice, definido como un sistema de tres componentes dinámicos (Universidad, Industria y Gobierno) que se van desarrollando en espiral para conseguir un objetivo común (ETZKOWITZ e LEYDESDORFF, 1998). De forma más concreta, el objetivo de esta investigación es realizar ese análisis en España.

La estructura del artículo es la siguiente: en primer lugar se desarrolla una revisión de la literatura que justifica la importancia del estudio y también la elección de las variables del modelo en función de investigaciones publicadas con anterioridad presentando las hipótesis a las que se pretende dar respuesta. En la tercera sección se presenta la metodología, la idoneidad de la misma con los datos utilizados y la descripción de las características de la muestra. La cuarta sección presenta el modelo propuesto y los resultados obtenidos, así como la interpretación de los mismos. Para finalizar se expone una breve conclusión sobre la importancia del estudio y la contribución que realiza, las limitaciones que se presentan y las posibles líneas de investigación futuras que se pueden seguir en relación a esto.

\section{REVISIÓN DE LITERATURA Y HIPÓTESIS}

El proceso de innovación se presenta, actualmente, como una herramienta estratégica para todas aquellas empresas que deseen crecer y mejor sus resultados (GARDAKER y otros, 1998). La innovación tecnológica es fuente de ventajas competitivas, lo que estimula la competitividad empresarial (CAMISON e VILLAR-LOPEZ, 2010), de ahí el gran interés que tiene para la sociedad actual lo tratado en este estudio.

Se define innovación en el ámbito de las organizaciones la creación de nuevo valor para los clientes y la empresa a través del cambio de una o más dimensiones en su sistema (SAWHNEY y otros, 2011). Para que este proceso se realice dentro de una organización, es necesario que posea la capacidad de utilizar el conocimiento existente para implementar nuevas ideas dentro de la misma (ZHAO y otros, 2005) de forma que desarrolle recursos tangibles e intangibles de forma continua (BARBIERI y otros, 2010). Por lo tanto, cuando se habla de un proceso de innovación en las empresas, se hace referencia a ideas creativas que marcan una diferencia con respecto a la competencia (MIRANDA e FIGUEIREDO, 2010).

Otro aspecto clave en el proceso de innovación de las empresas está relacionado con las fuentes de información (CRESPI y otros, 2008) puesto que su capacidad de innovación se ve influenciada positivamente por flujos externos de conocimiento (DIAZ DIAZ y otros, 2006). En este sentido, la heterogeneidad del conocimiento utilizado es identificada, en muchos casos como fundamental para el éxito en la innovación (AHUJA e LAMPERT, 2001; AHUJA e KATILA, 2004), esta heterogeneidad está relacionada con las distintas fuentes de información que pueden utilizar las empresas (VEUGELERS e CASSIMAN, 1999; AHUJA e KATILA, 2001; ROSENKOPF e NERKAR, 2001; LAURSEN e SALTER, 2006). Directivos y organizaciones aprenden y obtienen conocimiento de su entorno: clientes, proveedores, competidores, consultorías y otros agentes externos (WANG, 2009).

Esta importancia de las fuentes de información en la actividad innovadora de las empresas ha sido el eje fundamental de la investigación que se presenta, donde se analiza la relación entre resultados de innovación y la importancia que las organizaciones dan a la información obtenida por los agentes que configuran la Triple Hélice: Universidad, Industria y Gobierno (ETZKOWITZ e LEYDESDORFF, 1998).

La Triple Hélice es un modelo propuesto por los investigadores Loet Leydesdorff y Henry Etzkowitz que supone una orientación para las políticas de innovación (GONZALEZ DE LA FE, 2009) basado en tres ejes: industria, universidad y gobierno. Se considera prescriptivo porque impulsa el proceso y, simultáneamente, descriptivo porque recoge los casos donde la innovación ha tenido éxito (VIALE e GHIGLIONE, 1998). Sus autores lo desarrollan a partir de trabajos conjuntos y separados, actuando como consultores para aquellos países interesados en utilizar la innovación como herramienta de crecimiento (GONZALEZ DE LA FE, 2009), siendo su núcleo la expansión del conocimiento en la sociedad y la economía (ETZKOWITZ, 2002).

El eje industria, hace referencia a los negocios que conforman el tejido empresarial de un país. En el caso de la obtención de información de la actividad innovadora, estaríamos hablando de todas aquellas empresas que tienen relación con la 
organización que realiza el proceso de innovación, fundamentalmente proveedores y competidores.

Los proveedores están relacionados con la actividad innovadora de las empresas (GASSMANN y otros, 2010) y que las organizaciones expliciten en sus contratos con proveedores objetivos estratégicos de aprendizaje se relaciona con un efecto positivo en sus competencias internas (NORDBERG y otros, 2003). Además, si los proveedores participan en las primeras fases del proceso de innovación, el resultado del mismo podría verse incrementado (HAGEGOORN, 2002).

Los competidores son otro componente del eje industria. En general, la teoría económica ha relacionado la competitividad entre empresas con un mejor desempeño y mejores prácticas (NICKELL, 1996; OKADA, 2005). En el caso de la actividad innovadora, la competitividad entre empresas está ligada con mejores prácticas tecnológicas (BOLDRIN y otros, 2011). Este aspecto se encuentra relacionado con la orientación al competidor, definida como la habilidad de la empresa de responder ante las actividades que realiza su competencia (GATIGNON e XUEREB, 1997).

No solo la respuesta ante la competencia es un aspecto clave de la relación entre organizaciones, también es importante la cooperación entre ellas, especialmente en el caso de las pequeñas y medianas empresas (PYMEs) que perciben como una gran dificultad para la innovación el acceso a financiación (GARCIA-VEGA e LOPEZ, 2010). En este sentido, la colaboración se presenta como una útil herramienta que favorece el acceso a fuentes de financiación externas (QUINTANA e BENAVIDES, 2007) puesto que incrementa la percepción de probabilidades de éxito por parte de los inversores (STUART y otros, 1999).

En general, acuerdos de cooperación relacionados con la investigación y el desarrollo (I+D) potencian la tenencia de recursos necesarios para sobrevivir (TEECE, 2007). Por ejemplo Quintana y Benavides (2010) especifican en su investigación que en un sector específico las alianzas tecnológicas (en general acuerdos de cooperación en I+D) contribuyen al desarrollo de innovaciones radicales.

De esta forma se introduce el término coopetición (cooperación + competición), que hace referencia a la influencia en la actividad innovadora de los competidores en una empresa, tanto en relación a la competitividad como en el caso de la cooperación entre ellas (NALEBUFF e BRANDENBURGER, 1996).

En relación al eje industria expuesto, se propone la siguiente hipótesis:

$\mathrm{H}_{1}$ : Los proveedores y los competidores son fuentes de información para la innovación significativa dentro de la Triple Hélice.
El eje Universidad de la Triple Hélice hace referencia a la actividad que realizan las Universidades y Centros de Educación Superior de un país. Su contribución a la innovación empresarial se realiza a través del incremento de graduados e investigadores y el conocimiento que ofrece (MARTIN e TANG, 2007). Los estudios que han tratado de medir el efecto de la investigación en las Universidades públicas en la economía del país han mostrado una contribución positiva (ROOS e PIKE, 2011). Algunos de ellos muestran que: sobre el 10 por ciento de las innovaciones depende del apoyo de la investigación académica (MANSFIELD, 1991), el 20 por ciento de las innovaciones del sector privado se basan en investigaciones de Universidades públicas (TIJSSEN, 2002) y en la industria biomédica un incremento de 1 punto porcentual en la investigación académica conduce a un incremento de más de 2 puntos porcentuales en el número de compuestos disponibles comercialmente (TOOLE, 1999)

Sin embargo, estudios recientes muestran que ha habido una reducción de la cooperación en materia de innovación, entre empresas y Universidad, debido fundamentalmente a las barreras que perciben los directivos para colaborar con investigadores (BRUNEEL y otros, 2009)

En relación a todo lo expuesto acerca de las Universidades, se propone la siguiente hipótesis:

H2: La información que las empresas obtienen de Universidades y Centros de Enseñanza Superior refleja el eje Universidad de Triple Hélice.

El último de los ejes de la Triple Hélice es el conformado por el Gobierno. Los gobiernos juegan un papel recolector-benefactor apoyando la innovación en sus países de forma directa, y también de forma indirecta financiando en este caso Centros de Investigación (FLORICEL y otros, 2009). En relación a esto, se propone la siguiente hipótesis:

H3: La información para la innovación obtenida de Centros Tecnológicos y Organismos Públicos de Investigación refleja el eje Gobierno de la Triple Hélice.

Derivadas de las hipótesis anteriores, nos planteamos una relación causal entre la importancia que da la empresa a la información sobre innovación recibida de la Triple Hélice y sus resultados en innovación, que da lugar a las siguientes hipótesis:

H4: El nivel de importancia dado a la información recibida de la Triple Hélice para la innovación influye directamente en los resultados de innovación.

$\mathrm{H}_{5}$ : Los resultados de innovación inciden en el nivel de importancia que la organización da a la información que se obtiene de la Triple Hélice. 


\section{METODOLOGÍA}

\section{Base de datos}

La base de datos primaria de nuestra investigación está formada por los resultados agrupados de la encuesta anual sobre Innovación en las Empresas 2008-2010 que el Instituto Nacional de Estadística (INE) realiza a las organizaciones que desempeñan actividades de I+D+i (organizaciones EIN) en España. Dicha encuesta está integrada en los planes de estadística de la Unión Europea (UE).

Al centrarnos en resultados procedentes de la innovación y fuentes de información utilizadas para tomar decisiones sobre innovación, entendemos no son de aplicación los resultados del trabajo meta-analítico de Damanpur (1991) sobre el patrón innovador de las organizaciones, lo que justifica la decisión de trabajar con el universo propuesto.

Para esta fase de la investigación hemos optado por emplear datos agregados por actividad empresarial, que recogen las respuestas de 36.911 empresas (lo que dota al estudio del suficiente nivel de confianza) agrupadas por Clasificación Nacional de Actividades Económicas (CNAE). Esto da como resultado una muestra de 84 elementos, 47 corresponden a respuestas agrupadas por CNAE de grandes empresas y 47 a respuestas agrupadas por CNAE de pequeñas y medianas empresas. Se realiza esta distinción puesto que el acceso a recursos de innovación varía significativamente según el tamaño de empresa, lo que incide en los resultados del estudio.

La muestra obtenida cumple con los dos requisitos necesarios para la aplicación del modelo SEM (ecuaciones estructurales): muestra pequeña y número de observaciones algo superior al número de variables observadas multiplicado por diez (RUIZ y otros, 2010).

\section{Variables}

Nos hemos centrado en el constructo resultados de la encuesta estudiada y sus indicadores, y hemos definido un constructo con los indicadores que manifiestan el nivel de información que las organizaciones recogen de los agentes que integran la Triple Hélice. Entre las relativas a fuentes de mercado hemos excluido clientes (objeto de estudio aparte), debido a que su inclusión sesgaría sustancialmente los resultados de un estudio que se quiere centrar en los componentes más relevantes del eje industria de la Triple Hélice y, a su vez, menos conocidos y estudiados.

Los indicadores de resultados miden el porcentaje sobre la cifra total de negocio, y los relativos a las fuentes de información están operativizados mediante una escala Likert de 1 a 4 , por lo que son variables endógenas cuantitativas, lo que permite aplicar el modelo SEM basado en las correlaciones y covarianzas entre las variables de cada ecuación y las variables latentes explicadas. Su planteamiento en la encuesta sigue el procedimiento sugerido por Williams y otros (1989) para reducir cualquier efecto implícito en los entrevistados y mitigar el problema de varianza común.

El primer constructo, Impacto económico de las innovaciones sobre la cifra de negocios, trata de explicar el porcentaje que supone en la cifra de negocios total los ingresos debidos a actividades innovadoras.

Analizamos su relación con el constructo Fuentes agentes del Modelo Triple Hélice para actividades de innovación que trata de explicar en qué medida se tiene en cuenta, para tomar decisiones sobre innovación, la información suministrada por agentes del Modelo Triple Hélice. Los indicadores de ambos constructos se describen en Cuadro 1.

\section{CUADRO 1. Relación de variables con sus respectivos códigos}

\begin{tabular}{l|l|l|l}
\hline \multicolumn{2}{l|}{ Constructo o variable latente } & \multicolumn{2}{l}{ Variable explicativa } \\
\hline Código & Descripción & Código & Descripción \\
\hline \multirow{2}{*}{ CNNINN } & $\begin{array}{l}\text { Impacto económico de las } \\
\text { innovaciones sobre la cifra de negocios }\end{array}$ & CNNEMP & $\begin{array}{l}\text { Porcentaje debido a innovaciones en bienes y servicios introducidos en el } \\
\text { período 2008-2010 que únicamente fueron novedad para la empresa }\end{array}$ \\
\hline \multirow{2}{*}{ THELIX } & $\begin{array}{l}\text { Fuentes agentes del Modelo Triple } \\
\text { Hélice para actividades de innovación }\end{array}$ & INPROV & $\begin{array}{l}\text { Porcentaje debido a innovaciones en bienes y servicios introducidos } \\
\text { en el período 2008-2010 y que representaron una novedad para el } \\
\text { mercado en el que opera la empresa }\end{array}$ \\
\hline \multirow{2}{*}{} & & INCOOP & Competidores u otras empresas del mismo ramo de actividad \\
\cline { 2 - 4 } & & INFMAC & Universidad u otros centros de enseñanza superior \\
\cline { 2 - 4 } & & INFCCT & Consultores, laboratorios comerciales o institutos privados de I+D \\
\hline
\end{tabular}


La Tabla 2 muestra los estadísticos descriptivos de las variables explicativas así como sus correlaciones.

TABLA 1. Medias, desviaciones típicas y correlaciones de las variables explicativas

\begin{tabular}{|c|c|c|c|c|c|c|c|c|}
\hline Variable & Media & $\begin{array}{l}\text { Desviación } \\
\text { Típica }\end{array}$ & \multicolumn{6}{|c|}{ Correlaciones } \\
\hline CNNEMP & 14,60 & 5,97 & 1 & & & & & \\
\hline CNNMER & 11,74 & 8,22 & $0,580^{* *}$ & 1 & & & & \\
\hline INPROV & 8,14 & 7,25 & $0,458^{\star \star}$ & $0,449^{\star \star}$ & 1 & & & \\
\hline INFMAC & 2,51 & 3,09 & 0,214 & 0,253 & $0,631^{\star \star}$ & $0,627^{\star \star}$ & 1 & \\
\hline INFCCT & 2,24 & 2,01 & $0,332^{\star}$ & $0,324^{*}$ & $0,646^{\star *}$ & $0,640^{\star \star}$ & $0,629^{\star \star}$ & 1 \\
\hline
\end{tabular}

* La correlación es significativa al nivel o,05 (bilateral)

** La correlación es significativa al o,01

\section{Técnica de análisis}

Nos proponemos demostrar empíricamente la idoneidad de los indicadores para explicar los constructos planteados, así como la relación causal de interdependencia entre ambos constructos, para lo que hemos utilizado el paquete estadístico AMOS vr. 19. Para ello los modelos causales son los más indicados pues además de estimar los parámetros estructurales y proporcionar información completa, coherente y exacta de su validez, representan una relación teórica causa-efecto (PAGE, 1990), que se especifica en función de la teoría subyacente; de tal forma que, tal y como afirma Bizquerra (1989), su principal ventaja es pasar de una teoría expresada verbalmente a un modelo expresado matemáticamente.

El modelo causal adoptado ha sido el SEM, ya que nos permite estudiar las relaciones causales entre datos directamente observables, mediante la proposición del tipo y dirección de las relaciones esperadas y posterior estimación de los parámetros (BARRET, 2006) que vienen especificados por las relaciones propuestas a nivel teórico. De ahí que se denominen también análisis confirmatorios, pues su interés principal es confirmar mediante el análisis de la muestra las relaciones propuestas (JÖRESKOG, 1979).

En opinión de un buen número de autores como Fuentes (1985), Steiger (1990) o Austin y Calderón (1996), la metodología para validar este tipo de modelo causal consta de los siguientes pasos:

Paso 1. Especificación del modelo: mediante un esquema de la realidad observada y propuesta de las hipótesis mediante su representación gráfica (ver Figura 1).

Paso 2. Identificación del modelo: el modelo factorial confirmatorio estará identificado si todos los parámetros lo es- tán (BATISTA e COENDERS, 2000), es decir cada uno de los parámetros estimados tiene una única solución.

Como condición necesaria pero no suficiente hemos empleado la regla de los grados de libertad (df), obtenidos como la diferencia entre el número de varianzas y covarianzas y el número de parámetros a estimar. Si dfıo, el modelo está infra-identificado; si $\mathrm{df}=0$ el modelo está posiblemente identificado, y si df>o el modelo está sobre-identificado. En nuestro caso, $\mathrm{df}=8$ >o, por lo que el modelo está sobre-identificado.

Igualmente, una condición suficiente, aunque no necesaria es que sea recursivo, como en nuestro caso. Es decir, no contiene efectos circulares o recíprocos entre sus variables, luego está identificado.

Figura 1. Especificación del Modelo

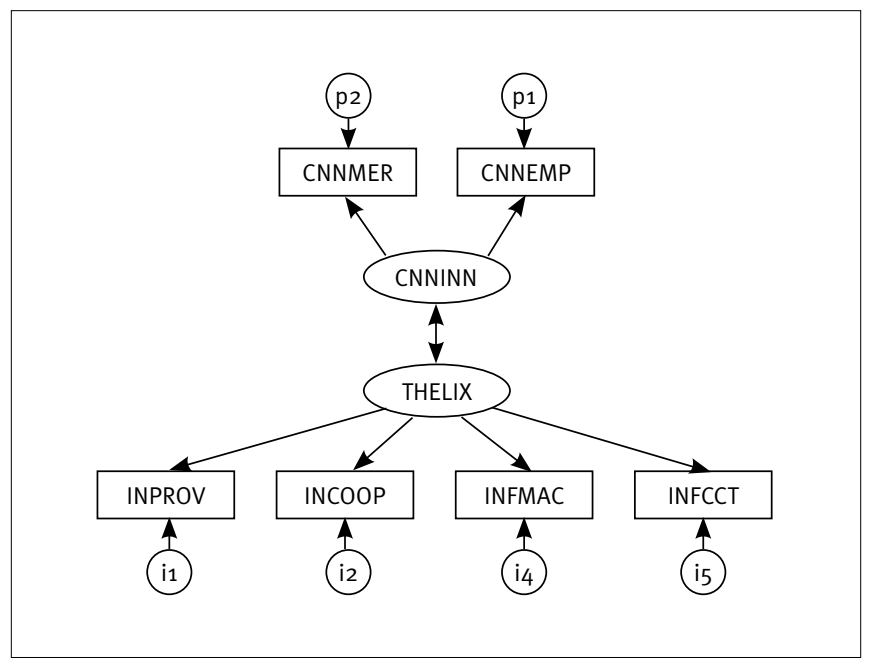

Paso 3. Estimación de parámetros: Los parámetros estimados son la magnitud de influencia que una variable "causa" provoca en la variable "efecto", las cuales se interpretan 
como pesos para efectos de análisis. Así, una vez obtenidos, los resultados han de examinarse minuciosamente, con el objeto de cotejar las relaciones causales que más sobresalgan por su peso y relevancia teórica (ACEVEDO, 2003).

Son las incógnitas del modelo estructural propuesto, que en nuestro caso, es un modelo factorial confirmatorio, cuya ecuación asociada es:

$X=\Lambda_{x} \zeta+\delta$

Los parámetros de este modelo son:

- Coeficientes de Wright $(\lambda)$ : son los pesos (cargas factoriales) de las variables endógenas de las ecuaciones estructurales del modelo y reciben el nombre de coeficientes de Wright. Constituyen la incógnita de la ecuación asociada. Su valor se determina resolviendo el sistema de ecuaciones estructurales del modelo (en nuestro caso seis, uno por variable observada)

- Variables residuales o errores ( $\delta)$ : representan los errores de medición o las variables que podrían influir en el modelo pero que no se encuentran incluidas en él. No se encuentran correlacionadas entre sí y cada variable residual o error ejerce influencia sobre una sola variable del modelo (en nuestro caso seis, uno por variable observada)

- Correlación simple de orden cero entre las variables latentes o constructos del modelo $(\Phi)$ : es el grado causa-efecto entre los constructos, nos da información sobre el grado en que, presumiblemente, variará un constructo ante una variación de aquél con el que está correlacionado (en nuestro caso una única correlación entre dos constructos planteados).

De entre todos los métodos de estimación de estos parámetros el iterativo de estimación de máxima verosimilitud (ML) resulta el más eficiente y no sesgado (HAYDUK, 1996) cuando se cumple el supuesto de normalidad multivariante o presenta ligeras oscilaciones respecto a ella.

Wang y otros (1996) aconsejan el método AGL, asintóticamente libre de distribución, debido a su insensibilidad respecto a la no normalidad de los datos. Este método exige un número considerable de casos ( $\mathrm{N}=500$ o más), por lo que, en nuestro caso, resulta inaplicable.

En nuestro estudio, dado el carácter pequeño de la muestra y la normalidad multivariada de los datos, hemos utilizado el método de máxima verosimilitud (ML).
Paso 4. Evaluación del modelo: la cuarta y última fase es la evaluación del modelo que se subdivide en dos: ajuste del modelo factorial confirmatorio y el ajuste del modelo en su globalidad.

El ajuste del modelo factorial confirmatorio reside en comprobar si existen coeficientes estimados que exceden los límites aceptables; es decir: (a) varianzas de error negativas o no significativas para algún constructo; (b) coeficientes estimados inferiores a 0,5 o superiores a 1; (c) errores de medida muy elevados (BENTLER, 1980; DILLON y otros, 1987), así como analizar la significación de los coeficientes estimados (nivel 0,05), de tal forma que cualquier parámetro estimado ha de ser estadísticamente distinto de cero.

La premisa del ajuste global del modelo es que existe un ajuste perfecto cuando hay una correspondencia perfecta entre la matriz reproducida por el modelo y la matriz de observaciones. Diferenciamos tres tipos de medidas dentro del ajuste global: (1) medidas absolutas de ajuste; (2) medidas incrementales de ajuste; y (3) medidas de ajuste de parsimonia.

(1) Las medidas absolutas establecen en qué medida el modelo predice la matriz de datos inicial. Las medidas generalmente aceptadas son: (a) $x^{2}$ (ji-cuadrado), aunque autores como Browne e Mels (1992, p. 78) indican “[...] la hipótesis nula, del ajuste perfecto, es implausible y no ayuda mucho a conocer si la prueba estadística hace algo para detectar que nuestro modelo es falso [...]". Sin embargo, es sensible, cuando trabajamos con muestras pequeñas, para comprobar el tamaño de la muestra y la no normalidad en la distribución de las variables de entrada de datos; (b) $\mathrm{x}^{2} / \mathrm{df}$ (ji-cuadrado relativa donde $\mathrm{df}=$ grados $\mathrm{de}$ libertad) proporciona un coeficiente comprendido por el mínimo de la discrepancia dividido por los grados de libertad; cuanto más pequeño más calidad en el ajuste del modelo (WHEATON y otros, 1977; MARSH e HOCEVAR, 1985); (c) RMSEA (error de aproximación de la raíz media cuadrática) divide la discrepancia entre los grados de libertad al cuadrado. Esto quiere decir que la discrepancia se calcula tanto en términos de la muestra utilizada para la estimación como en términos de población (BROWNE e CUDECK,1993); (d) PCLOSE es un p valor que prueba la hipótesis nula de la cercanía del ajuste del RMSEA; (e) ECVI (índice de validación cruzada esperada) que estima una aproximación a la bondad de ajuste que alcanzaría el modelo estimado en otra muestra del mismo tamaño, teniendo en cuenta el número de parámetros estimados para el modelo de global (DIAZ e BEERLI, 2006); (f) FMIN (valor mínimo de la función de discrepancia) cuya ventaja es que no se ve afectado por el tamaño de la muestra, (g) $F_{M L}$ (valor estimado de la función de discrepancia) es el valor de la función de discrepancia que se obtiene por el ajuste del modelo utilizando una estimación de la población en lugar de la muestra (STEIGER y otros, 1985). 
(2) Las medidas descriptivas (ajuste incremental), basadas en la comparación de modelos, comparan el modelo propuesto con el peor modelo posible, siendo el modelo nulo el más utilizado pues en él se supone que no hay relación entre las variables (HU e BENTLER, 1999). Las medidas normalmente utilizadas son: (a) TLI o índice de Tucker y Lewis; (b) NFI (índice de ajuste normado) que evalúa la reducción proporcional en la función de ajuste cuando se pasa del modelo nulo al propuesto (BENTLER e BONNET, 1980); (c) RFI (índice de ajuste relativo) que se obtiene sustituyendo en el NFI F/d por F; (d) CFI (índice de ajuste comparado) aporta información sobre la pérdida relativa de ajuste del modelo propuesto frente a la pérdida de ajuste del modelo nulo; (e) IFI (índice incremental de ajuste)

(3) Las medidas de parsimonia muestran el grado en que alcanza ajuste para cada coeficiente estimado y sirven de criterio de selección entre modelos alternativos. Un buen ajuste de parsimonia del modelo asegura que los resultados se mantendrán si analizamos otra muestra. Las medidas habituales son: (a) PNFI (índice de ajuste parsimonioso normado) resultado de la multiplicación del NFI por un índice de parsimoniosidad, tomando en cuenta el número de grados de libertad para probar el modelo que va a ser evaluado y el modelo nulo (JAMES y otros, 1982; MULAIK y otros, 1989); (b) PCFI (índice de ajuste parsimonioso comparado) resultado de la multiplicación del CFI, por un índice de parsimoniosidad, tomando en cuenta el número de grados de libertad para probar el modelo que va a ser evaluado y el modelo (JAMES y otros, 1982; MULAIK y otros, 1989); (c) PRATIO (razón de parsimoniosidad) expresa el número de restricciones en el modelo que va a ser evaluado como una fracción del número de restricciones en el modelo independiente (HU e BENTLER, 1999); (d) Hoelter (1983) indica que se considera adecuado el tamaño de una muestra si su valor es superior a 200 e inaceptable si es inferior a 75, tanto para nivel de significación 0,05 como para un nivel de significación de 0,01.

En la Tabla 2 presentamos un resumen de las medidas, sus fórmulas y sus valores adecuados.

\section{TABLA 2. Medidas de ajuste global}

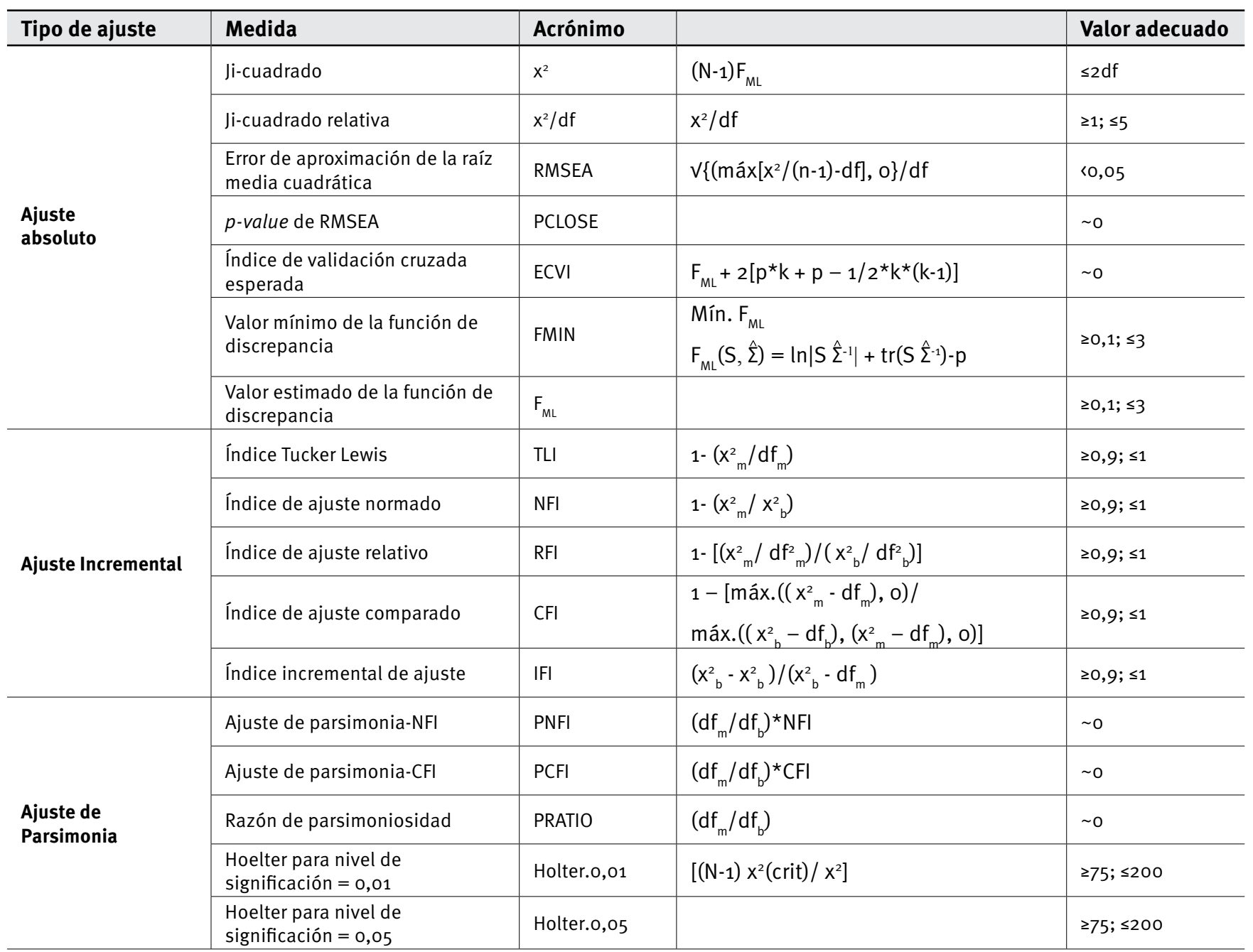




\section{RESULTADOS Y INTERPRETACIÓN}

\section{Fiabilidad y normalidad de escala}

Para medir la fiabilidad de las variables explicativas utilizamos el alpha de Cronbarch (ver Tabla 3), estadístico que supone un modelo de consistencia interna que estima el límite inferior del coeficiente de fiabilidad basándose en el promedio de las correlaciones entre las variables observadas. Realizamos la medición para el conjunto de variables y para cada grupo de indicadores. Los resultados obtenidos, utilizando la aplicación SPSS vr. 19 son un a de 0,80 para el total de variables, un a de 0,71 para las variables del constructo $\mathrm{CN}$ NINN y un a de 0,82 para las del constructo THELIX. Las variables superan la prueba de fiabilidad, al dar valores superiores a 0,70 (NUNNALLY, 1978), por lo que podemos continuar nuestro estudio.

Aplicamos el coeficiente de Mardia para comprobar la normalidad multivariante (ver Tabla 3) (BOLLEN, 1989) y vemos que es inferior a $p^{\star}(p+2)$, donde $p$ es el número de variables observadas, por lo que existe normalidad multivariada

Los resultados obtenidos nos indican la conveniencia del método de estimación de máxima verosimilitud $(\mathrm{ML})$ en nuestro análisis.

\section{TABLA 3. Fiabilidad y Normalidad}

\begin{tabular}{|c|c|c|}
\hline \multicolumn{3}{|l|}{ Alfa de Cronbach } \\
\hline \multirow[t]{2}{*}[\mathrm{k}/(\mathrm{k}-1)]{$\left[1-\left(\sum \mathrm{s}_{\mathrm{i}}^{2} / \mathrm{s}_{\text {sum }}^{2}\right)\right.$} & CNNINN & 0,71 \\
\hline & THELIX & 0,82 \\
\hline & $P(p+2)$ & 48,000 \\
\hline$(1 / n) \sum_{i=1}^{n}\left\{\left(x_{i}-x\right)\right\}^{2}$ & Coeficiente & 35,137 \\
\hline
\end{tabular}

\section{Modelo de Factorial Confirmatorio}

El modelo factorial confirmatorio nos permite explicar la correlación entre constructos así como la asociación entre cada constructo y sus correspondientes variables observadas, mediante el cálculo de sus cargas factoriales.

En nuestro caso, hemos planteado dos constructos con sus correspondientes variables observadas, cuya validez pretendemos confirmar con la aplicación de este modelo.
En primer lugar comprobamos que los indicadores se adecúan a los constructos. Al ser modelos basados en estructuras de covarianzas cuentan con índices que permiten evaluar los componentes de la estructura factorial del modelo (SCHUMACKER e LOMAX, 2004) que requiere de cargas factoriales superiores a 0,5 e inferiores a<1,0.

En nuestro caso las cargas (ver Tabla 4) reflejan los constructos que representan; los errores de medida no son elevados y no aparecen varianzas de error negativas o no significativas, lo que valida el path-diagram del Figura 2.

\section{TABLA 4. Pesos de regresión estandarizados y sus errores de medida}

\begin{tabular}{|c|c|c|c|c|c|}
\hline Indicadores & \multicolumn{2}{|c|}{ Constructos } & Estimación & Error & Varianza del error \\
\hline CNNMER & $<-$ & CNNINN & 0,755 & 0,57 & 28,38 \\
\hline INFCCT & $<--$ & THELIX & 0,771 & 0,59 & 3,59 \\
\hline INFMAC & $<--$ & THELIX & 0,683 & 0,47 & 1,61 \\
\hline INPROV & $<-$ & THELIX & 0,964 & 0,33 & 2,26 \\
\hline
\end{tabular}


El modelo incluye posibles relaciones causales entre constructos. En nuestro caso, y tal y como se puede apreciar en el Figura 2, el modelo muestra una relación causal directa entre ambos constructos con un nivel alto de correlación.

\section{Figura 2. Modelo Global de Ecuaciones Estructurales}

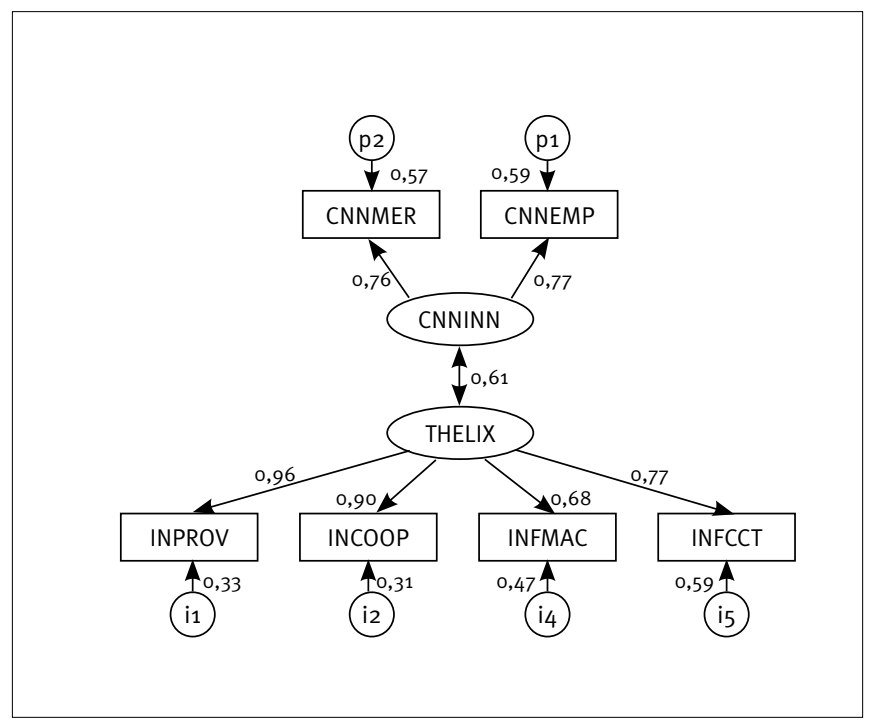

Evaluamos el ajuste del modelo a través del análisis de significación de los coeficientes estimados. Para ello revisamos la proporción crítica (C.R.), que es el cociente entre la estimación del parámetro y la estimación del error estándar. Si se reúnen las suposiciones de distribución apropiadas, cuando a una estimación le corresponde una proporción crítica mayor que 2 en valor absoluto, el parámetro es significativamente diferente de cero al nivel ,05 y el modelo presentará un buen ajuste.

En nuestro caso, el valor más pequeño encontrado es de 3,17 , por lo que podemos concluir que el ajuste es bueno, hecho que valida el modelo factorial confirmatorio propuesto, cuyas ecuaciones son:

$$
\begin{aligned}
& \text { CNNMER }=0,76 \text { CNNINN }+0,57 \\
& \text { CNNEMP }=0,77 \text { CNNINN }+0,59 \\
& \text { INPROV }=0,96 \text { THELIX }+0,33 \\
& \text { INCOOP }=0,90 \text { THELIX }+0,31 \\
& \text { INFMAC }=0,68 \text { THELIX }+0,47 \\
& \text { INFCCT }=0,77 \text { THELIX }+0,59
\end{aligned}
$$

\section{Modelo global: sus ajustes}

En este apartado determinamos las medidas de ajuste global para establecer el grado en que el modelo predice la matriz de datos inicial. El modelo es recursivo y consta de 6 indicadores para 2 constructos (dimensiones), los grados de libertad son 8 y el número de parámetros estimados es 13 . El resultado de los ajustes del modelo es bueno salvo la parsimoniosidad (ver Tabla 5).

\section{TABLA 5. Resultados de los ajustes del modelo}

\begin{tabular}{c|c|c|c|c|c|c|c|c}
\hline \multicolumn{3}{c|}{ Ajuste absoluto } & \multicolumn{3}{c|}{ Ajuste incremental } & \multicolumn{3}{c}{ Ajuste de parsimonia } \\
\hline Medida & Valor & Nivel & Medida & Valor & Nivel & Medida & Valor & Nivel \\
$\mathrm{X}^{2}$ & 14,02 & Muy Bueno & $\mathrm{TLI}$ & 0,92 & Bueno & PNFI & Malo \\
\hline $\mathrm{X}^{2} / \mathrm{df}$ & 1,75 & Muy Bueno & $\mathrm{NFI}$ & 0,91 & Bueno & PCFI & 0,51 & Malo \\
\hline RMSEA & 0,13 & Marginal & RFI & 0,84 & Marginal & PRATIO & 0,53 & Malo \\
\hline PCLOSE & 0,12 & Marginal & CFI & 0,96 & Muy Bueno & Hoelter 0,01 & 46,00 & Malo \\
\hline ECVI & 1,27 & Malo & IFI & 0,96 & Muy Bueno & Hoelter 0,05 & 59,00 & Malo \\
\hline FMIN & 0,34 & Bueno & & & & & & \\
\hline$F_{\text {ML }}$ & 0,15 & Bueno & & & & &
\end{tabular}

\section{Resultados}

Desechamos la variable fuentes institucionales organismos públicos de investigación por presentar una correlación excesiva con el resto de variables explicativas de fuentes institucionales. En nuestra opinión este resultado se debe a que la pregunta se refiere a Organismos Públicos de Investigación, entidades que muchos de los encuestados pueden inferir que son Centros de Enseñanza Superior y Centros Tecnológicos; esto es, estaría respondida en ellas. 
El modelo de medida presenta cargas factoriales (pesos) superiores a 0,5 e inferiores a 1 para todos los indicadores por lo que resulta válido y los indicadores son idóneos para sus constructos.

Los indicadores muestran cargas factoriales altas o muy altas, por lo que la calidad explicativa del constructo es alta, lo que nos confirma las hipótesis H.1, H.2 y H.3. Esto es, las empresas reconocen los ejes Industria, Universidad y Gobierno como importantes fuentes de información sobre innovación.

Cabe destacar los valores alcanzados por las variables INCOOP e INPROV, que avala las teorías sobre la coopetición, que fija como uno de los factores clave para la competitividad, especialmente en PYMES, la cooperación con competidores y proveedores

Asimismo, se aprecia que la variable con menor peso es INFMAC, este hecho refleja que, aun conscientes de que estos Centros son espacios de creación y almacenamiento de conocimiento, tan necesario para la innovación, el contacto Universidad-Empresa sigue sin ser suficientemente fluido. Esta evidencia indica la necesidad de seguir impulsando lugares de encuentro (redes, clusters...) en los que afianzar y explotar las ventajas que ofrece la Triple Hélice en la generación de innovación.

La significativa correlación entre constructos nos indica que la generación de Capital Relacional con agentes de la Triple Hélice está directamente relacionada con los resultados procedentes de la innovación; y, a su vez, estos resultados influyen en el nivel de importancia que se da a la información procedente de la Triple Hélice.

En cuanto al ajuste, la bondad del modelo y las medidas incrementales ofrece muy buenos resultados. Por tanto, el modelo propuesto se aproxima en gran medida a la realidad. Este hecho junto a lo expuesto en el párrafo anterior confirma H.4 y H.5.

Sin embargo, dado que los índices relativos al ajuste de parsimonia se alejan de los valores recomendados no podemos afirmar que el modelo siga idéntico comportamiento si analizamos otra muestra.

\section{CONCLUSIONES}

En la sociedad actual la innovación se presenta como un recurso esencial. Este trabajo muestra la relación existente entre los resultados de innovación de las empresas españolas durante los años 2008-2010 y la importancia que los directivos de las mismas dan a la información obtenida por los agentes que conforman la Triple Hélice.

Esta investigación manifiesta que, en el caso de las empresas españolas, los tres ejes de la Triple Hélice son importan- tes para los resultados de innovación. A su vez, los resultados de innovación, influyen en la importancia que dan los directivos de las empresas a la información que obtienen de cualquiera de los vértices de la Triple Hélice.

Por lo tanto se demuestra la interrelación existente entre resultados de innovación de las empresas y la información que obtienen a través de la Triple Hélice. Esto es muy interesante puesto que es un estudio empírico que demuestra esta teoría en el ámbito de la innovación, siendo especialmente relevante el indicador relacionado con Centros Tecnológicos Institucionales puesto que no es un aspecto que haya sido muy desarrollado en la literatura de innovación.

En base a lo anterior, las implicaciones de esta investigación son diversas:

Por un lado, se presenta como un punto de partida para nuevas aportaciones y estudios en ámbito académico dedicado a la innovación de las empresas. En esta área, las publicaciones relacionadas con la Triple Hélice no son muchas y se destaca la importancia de estos tres ejes que se pueden abordar en futuras investigaciones.

En relación con las empresas interesadas en mejorar su actividad innovadora, este trabajo supone un primer paso para que conozcan algunos aspectos importantes e interesantes relacionados con los resultados de innovación. De esta forma, las empresas conocen la importancia de establecer relación con otros miembros de la Triple Hélice.

Finalmente se identifica una implicación para los gobiernos y administraciones públicas, destacando el interés de su colaboración con las empresas y otros agentes en la actividad innovadora de las organizaciones privadas.

En cuanto a las limitaciones del análisis, cabe destacar aquellas derivadas de la obtención de los datos por parte del INE, puesto que la disponibilidad de datos para realizar el análisis empírico ha condicionado los resultados.

En futuras investigaciones sería muy interesante estudiar con más detenimiento cada uno de los ejes de la Triple Hélice por separado con el objetivo de obtener un mayor detalle en los resultados.

\section{REFERENCIAS}

ACEVEDO, R. Factores que inciden en la competencia docente universitaria. 2003. Tesis de Doctoral ed. Madrid: Universidad Complutense de Madrid, Madrid, 2003.

AHUJA, G; KATILA, R. Technological acquisitions and the innovation performance of acquiring firms: a longitudinal study. Strategic Management Journal, v. 22, n. 3, p. 197-220, 2001.

AHUJA, G; KATILA, R. Where do resources come from? The role of idiosyn- 
cratic situations. Strategic Management Journal, v. 25, n. 8-9, p. 887907, 2004.

AHUJA, G; LAMPERT, C. Entrepreneurship in the large corporation: a longitudinal study of how established firms create breakthrough inventions. Strategic Management Journal, v. 21, n. 6-7, p. 521-543, 2001.

AUSTIN, J; CALDERON, R. Theorical and technical contributions to structural equations modelling: an updated bibliography. Structural Equation Modeling, v. 3, n. 2, p. 105-125, 1996.

BARBIERI, J; VASCONCELOS, I; ANDREASSI, T; VASCONCELOS, F. Inovação e sustentabilidade: novos modelos e proposições. RAE-Revista de Administração de Empresas, v. 50, n. 2, p. 146-154, 2010.

BARRET, P. Structural equation modeling: adjudging model fit. Science Direct.Personality and Individual Differences, v. 42, n. 5, p. 815-824, 2006.

BATISTA FOGUET, J; COENDERS GALLART, G. Modelos de ecuaciones estructurales. Cuadernos de Estadística 6 ed. Madrid, España: La Muralla, 2000.

BENTLER, P. Multivariate analysis with latent variables: causal modeling. Annual Review of Psychology, v. 31, p. 419-456, 1980.

BENTLER, P; BONNET, D. Significance test and goodness of fit in the analysis of covariance structures. Psychological Bulletin, v. 88, n. 3, p. 588-606, 1980.

BIZQUERRA, R. Introducción conceptual al análisis multivariante. Un enfoque informático con los paquetes SPSS-X, BMDP, LISREL y SPAD. Barcelona: PPU, 1989.

BOLDRIN, M; CORREA-ALLAMAND, J; LEVINE, D; ORGANHI, C. Competition and innovation. Cato Papers on Public Policy, v. 1, p. 2-62, 2011.

BOLLEN, K. Structural equations with latent variables. New York: J. Wiley \& Sons, 1989.

BROWNE, M; CUDECK, R. Alternative ways of assessing model fit. En: K. Bollen \& J. Long, edits. Testing structural equation models. Newbury Park, California: Sage, p. 136-162, 1993.

BROWNE, M; MELS, G. RAMONA user's guide. Ohio: department of Psychology, Ohio State University, 1992.

BRUNEEL, J; D`ESTE, P; NEELY, A; SALTER, A. The search for talent and technology: Examining the Attitudes of EPSRC Industrial Collaborators towards Universities. Londres: Advanced Institute of Management Research, 2009.

CAMISÓN, C; VILLAR-LÓPEZ, A. Análisis del papel mediador de las capacidades de innovación tecnológica en la relación entre la forma organizativa flexible y el desempeño organizativo. Cuadernos de Economía y Dirección de la Empresa, v. 13, n. 45, p. 115-144, 2010.

CHEN, D; DALHMAN, C. Knowledge and development: a cross-section approach. World Bank Policy Research, p. Working paper. 3366, 2004.

CRESPI, G; CRISCUOLO, C; HASKEL, J. Productivity, exporting and the learning-by-exporting hypothesis: Direct evidence from UK Firms. Canadian Journal of Economics, v. 41, n. 2, p. 619-637, 2008.

DAMANPOUR, F. Organizational innovation: a meta-analysis of effects of determinants and moderators. Academy of Management Journal, v. 34, n. 3, p. 555-590, 1991

DÍAZ DÍAZ, N; AGUIAR DÍAZ, I; DE SAÁ PÉREZ, P. El conocimiento organizativo tecnológico y la capacidad de innovación. Evidencia para la empresa industrial española. Cuadernos de Economía y Dirección de la Empresa, v. 27, p. 33-60, 2006.
DÍAZ, G; BEERLI, G. El proceso de adopción de la conducta de reciclado: modelos explicativos y variables modeladoras. Cuadernos de Economía y Dirección de la Empresa, v. 28, p. 55-86, 2006.

DILLON, W; KUMAR, A; MULANI, N. Offending estimates in covariance structure analysis: comments on the causes of and solutions to Heywood cases. Psychological Bulletin, v. 101, n. 1, p. 126-135, 1987.

ETZKOWITZ, H. Networks of innovation: Science, technology and development in the Triple Helix Era. International Journal of Technology Management and Sustainable Development, v. 1, n. 1, p. 7-20, 2002.

ETZKOWITZ, H; LEYDESDORFF, L. The Triple Helix as a Model for Innovation Studies. Science \& Public Policy, v. 25, n. 3, p. 195-203, 1998.

FLORICEL, S; MICHELA, J; GEORGE, M. Resource feedbacks for continuous innovation: the articulation of firm, university and government roles. Copenhagen, Dinamarca, Summer Conference, Copenhagen Business School (CBS), 2009.

FUENTES, A. Los modelos causales en la investigación del rendimiento académico. Gijón, España, III Seminario sobre Modelos de Investigación Educativa, 1985.

GARCÍA-VEGA, M; LÓPEZ, A. Determinants of abandoning innovative activities: evidence from Spanish Firms. Cuadernos de Economía y Dirección de la Empresa, v. 13, n. 45, p. 69-92, 2010.

GARDAKER, G; AHMED, P; GRAHAM, G. An integrated response towards the pursuit of fast time to market of NPD in European manufacturing organizations. European Business Review, v. 98, n. 3, p. 172-177, 1998.

GASSMANN, O; ENKEL, E; CHESBROUGH, $\mathrm{H}$. The future of open innovation. R\&D Management, v. 40, n. 3, p. 213-221, 2010.

GATIGNON, H; XUEREB, J. Strategic orientation of the firm and new product performance. Journal of Marketing Research, v. 34, n. 1, p. 77-79, 1997.

GONZÁLEZ DE LA FE, T. El modelo de Triple Hélice de relaciones Universidad, industria y Gobierno: un análisis crítico. ARBOR Ciencia, Pensamiento y Cultura,v. CLXXXV, n. 738, p. 739-755, 2009.

HAGEDOORN, J. Inter-firm R\&D partnerships: an overview of major trends and patterns since 1960. Research Policy, v. 31, n. 4, p. 477-492, 2002.

HAYDUK, L. LISREL issues, debates and strategies. Baltimore: JHU Press, 1996.

HOELTER, J. The analysis of covariance structures: goodness of fit indices. Sociological Methods and Research, v. 11, n. 3, p. 325-344, 1983.

HU, L; BENTLER, P. Cutoff criteria for fit indexes in covariance structure analysis: Conventional criteria versus new alternatives. Structural Equation Modeling, v. 6, n. 1, p. 1-55, 1999.

JAMES, L; MULAIK, S; BRETT, J. Causal analysis: assumptions, models and data. Beberly Hills: Sage-Johns Hopkings University Press, 1982.

JÖRESKOG, K. A general approach to confirmatory maximum likelhood factor analysis with addendum. En: K. J. \&. D. Sörbom, ed. Advances in factor analysis and structural equation model. Cambridge: Abt Books, 1979.

LAURSEN, K; SALTER, A. Open for innovation: the role of openness in explaining innovative performance among UK manufacturing firms. Strategic Management Journal, v. 27, n. 2, p. 131-150, 2006.

MANSFIELD, E. Academic research and industrial innovation. Research Policy, v. 20, n. 1, p. 1-12, 1991.

MARSH, H; HOCEVAR, D. Application of confirmatory factor analysis to the study of self-concept: first and higher order factor models and their invariance across groups. Psychological Bulletin, v. 97, n. 3, p. 562-582, 1985. 
MARTIN, B; TANG, P. The benefits from publicly funded research. SPRU Electronic Working paper, v. 41, 2007.

MIRANDA, E; FIGUEIREDO, P. Dinâmica da acumulaçao de capacidades inovadoras: evidências de empresas de software no Rio de janeiro e em Sao Paulo. RAE-Revista de Administração de Empresas, v. 50, n. 1, p. 75-93, 2010.

MULAIK, S; JAMES, L.R; VAN ALSTINE, J; BENNETT, N; LIND, S; STILWELL, C. Evaluation of goodness of fit indices for structural equation models. Psychological Bulletin, v. 105, n. 3, p. 430-445, 1989.

NALEBUFF, b; BRANDENBURGER, A. Coopetition - kooperativ konkurrieren. Frankfurt, Mit der Spieltheorie zum Unternehmenserfolg, 1996.

NICKELL, S. Competition and corporate performance. Journal of Political Economy, v. 104, n. 4, p. 724-746, 1996.

NORDBERG, M; CAMPBELL, A; VERBEKE, A. Using customer relationships to acquire technological innovation: a value-chain analysis of supplier contracts with scientific research institutions. Journal of Business Research, v. 56, n. 9, p. 711-719, 2003.

NUNNALLY, J. Physhometric theory. $2^{\mathrm{a}}$ ed. New York: McGraw-Hill, 1978.

OKADA, Y. Competition and productivity in Japanese manufacturing industry. NBER Working paper, Issue no. 11540, 2005.

PAGE, A. Hacia un modelo causal del rendimiento académico. Madrid: CIDE, 1990.

QUINTANA, C.; BENAVIDES, A. Concentraciones territoriales, alianzas estratégicas e innovación. Un enfoque de capacidades dinámicas. Cuadernos de Economía y Dirección de la Empresa, v. 10, n. 30, p. 5-37, 2007.

QUINTANA, C.; BENAVIDES, A. Relación tecnológica en los acuerdos de cooperación empresarial y generación de innovaciones. Cuadernos de Economía y Dirección de la Empresa, v. 13, n. 45, p. 43-68, 2010.

ROOS, G; PIKE, S. The relationship between University research and firm innovation. En: E. Evans, R. Burritt \& J. Guthrie, edits. Bridging the gap between academic accounting research and professional practice. s.l.:The Institute of Chartered Accountants in Australia and Centre for Accounting, Governance and Sustainability, University of South Australia, p. 31-50, 2011.

ROSENKOPF, L; NERKAR, A. Beyond local search: boundary-spanning, exploration and impact in the optical disk industry. Strategic Management Journal, v. 22, n. 4, p. 287-306, 2001.

RUIZ, M; PARDO, A; SAN MARTIN, R. Modelos de ecuaciones estructurales. Papeles del Psicólogo, v. 31, n. 1, p. 34-45, 2010.

SAKAIYA, T. The knowledge value revolution or a History of the future. Tokio: Kodansha, 1991.

SAWHNEY, M; WOLCOTT, R; ARRONIZ, I. The different ways for companies to innovate. Sloan Management Review, v. 47, n. 3, p. 28-34, 2006.

SCHUMACKER, R; LOMAX, R. A begginer's guide to structural equation modeling. 2 ed. Mahwah: Lawrence Eribaum Soociates, 2004.
STEIGER, J. Structural model evaluation and modification: an interval estimation approach. Multivariate Behavioral Research, v. 25, n. 2, p. 173-180, 1990.

STEIGER, J; SHAPIRO, A; BROWNE, M. On the multivariate asymptotic distribution of sequential chi-square statistics. Psychometrika, v. 50, n. 3, p. 253-263, 1985.

STUART, T; HOANG, H; HYBELS, R. Interorganizational endorsements and the performance of entrepreurial ventures. Administrative Science Quarterly, v. 44, n. 2, p. 315-349, 1999.

TEECE, D. Explicating dynamic capabilities: the nature and microfoundations of (sustainable) enterprise performance. Strategic Management Journal, v. 18, n. 13, p. 1319-1350, 2007.

TIJSSEN, R. Science dependence of technologies: evidence from inventions and their inventors. Research Policy, v. 31, n. 4, p. 509-526, 2002.

TOOLE, A. The impact of federally funded basic research on industrial innovation: evidence from the pharmaceutical industry. Standford Institute for Economic Policy Research, Issue SIEPR Discussion Paper No. 98-8, 1999.

VASCONCELOS, G; WILKINSON, J; AMÂNCIO, R. Empreendedorismo, inovaçao e redes: uma nova abordagem. RAE-eletrônica, v. 7, n. 1, 2008. Disponible en: http://rae.fgv.br/sites/rae.fgv.br/files/artigos/10.1590_ S1676-56482008000100008.pdf. Acesso en 20.12.2012.

VEUGELERS, R; CASSIMAN, B. Make and buy in innovation strategies: evidence from Belgian manufacturing firms. Research Policy, v. 28, n. 1, p. 63-80, 1999.

VIALE, R; GHIGLIONE, B. The Triple Helix model: a tool for the study o European Regional Socio Economic Systems. The IPTS Report, European Commission, v. 29, 1998.

WANG, L; FAN, X; WILSON, V. Effects of nonnormal data on parameter estimates for a model with lateng and manifiest variables: an empirical study. Structural Equation Modeling, v. 3, n. 3, p. 228-247, 1996.

WANG, P. Popular concepts beyond organizations: exploring new dimensions of information technology innovations. Journal of the Association for Information Systems, v. 10, n. 2, p. 1-30, 2009.

WHEATON, B; MUTHÉN, B; ALWIN, D; SUMMERS, G. Assessing reliability and stability in panel models. En: D. Heise, ed. Sociological Methodology. s.l., p. 84-136, 1977.

WILLIAMS, L; COTE, J; BUCKLEY, M. Lack of method variance in self-reported affect and perceptions at work: reality or artefact?. Journal of Applied Psychology, v. 3, n. 74, p. 462-488, 1989.

ZHAO, H; TONG, X; WONG, P; ZHU, J. Types of technology sourcing and innovative capability: an exploratory study of Singapore manufacturing firms. Journal of High Technology Management Research, v. 16, n. 2, p. 209-224, 2005. 